\title{
The Research of Knowledge Workers' Competencies Based on EEG Experiment of Mental Operations
}

\author{
Huan $\mathrm{Cao}^{1}$, Yongjian $\mathrm{Li}^{2}$ \\ ${ }^{1}$ School of Management and Economics, University of Electronic Science and Technology of China, Chengdu, China; ${ }^{2}$ School of \\ Economics and Management, Southwest Jiaotong University, Chongqing, China. \\ Email: caohuan@uestc.edu.cn
}

Received September $12^{\text {th }}, 2009$; revised October $28^{\text {th }}, 2009$; accepted December $10^{\text {th }}, 2009$.

\begin{abstract}
The paper analyzes the limitation of the method to study the competencies. The differences between competencies of knowledge workers exist in the "therbligs" used in information process. Based on 191 questionnaires, advance the 9 common mental operations: metacognition, distinguishing, memory, transforming, imagining, character extracting, character integrating, reasoning and concretization. And the metacognition plays a role on planning, supervising and adjusting to the other mind operations. Then the paper designs a mental arithmetic experiment to compare the difference in the brain electric power spectrum and the brain electronic topographic diagram between the subjects of the different performance. And the paper further discusses the feasibility of the method to explore the competencies.
\end{abstract}

Keywords: Mental Therbligs, EEG, Knowledge Workers, Competencies

\section{Introduction}

In today's competitive business world, sustainable competitive advantage is the fundamental factor for organizations to survive. Many organizations have found that the ability of their workforce is the key to gain competitive advantage. For organizations to perform effectively, they need system to assess and develop employee performance against conceptually sound and measurable criteria, because it is necessary for an organization to know whether its people possess the abilities critical for success to adapt to changing demands [1]. An important milestone for clarifying the requirements for successful performance is the competency approach. The set of competencies usually serves as a platform for various HR practice such as performance evaluation, compensation, selection, and training [2].

The study of competency could be traced back to the Taylor's "motion-time" study. According to the Management Competencies Movement, Taylor became the first scholar who explored the competency systematically. McClelland published the article Testing for Competency rather than Intelligence which indicates the beginning of the modern competency movement.

Today, due to the nature of work shifting into the mental labor, the research on the competency should not depend on the motion-time analysis any more, but turn to job analysis, and should analyze it rationally [3-5].

The competency attracts a lot of interested among the scholars of management, psychology and human resource. And the research could be divided into 3 fields: differential psychology, education and behavior, and organizational psychology [6].

There are two common approaches to competencies: the US approach and the UK approach. Boyatzis, who exemplifies the US approach, define competency broadly as " an underlying characteristics of a person". It could be a "motive, trait, skill, aspect of one's self-image or social role, or a body of knowledge which he or she uses". The UK Government Employment Department defined competencies more broadly. This approach identified the outcome expected from a job when it is performed adequately. Day's definition of competence "the ability to put skills and knowledge into action" is an apt description of the UK approach.

There are many methods to study the competency.

McClelland [7] advanced the Behavioral event interview (BEI). Shi [8] using the BEI set up the competency model about the manager in telecom in China. And Shi [9] set up the competency model of the managers in family business in China. 
Folch, Lyon and Trost [10] put forward the focus group session which required the excellent performance employee to retrospect, discuss the duty and requirement of the work, and infer the trend in the future.

Sandberg [11] used the phenomenography to study the competency of engineers in Volvo. Through the interview, he concluded that what kind of competency will be developed is accord with the worker's own concept of work itself.

Chen [12] uses the text analysis and phone interview to develop the questionnaire of Chinese HR managers' competency.

Luo [13] used the Delphi to explore the military commander students' competency.

Comparing the different methods, Zhong [6] concluded, the picture story exercise, focus group session and systematic multiple level observation of groups are the most effective method to study the competency of manager.

In spite of the flourishing popularity of competency research, there exists the limitation as follows:

1) Until now, there is no unified definition of competency [14]. According to Bradley, our ways of thinking and describing competence are "primitive and clumsy"; for all the definitions and articles, "few are certain in their own minds what it means". As the concept matured, literatures focus specific view of competencies on "knowledge, skills, abilities, or other characteristics (KSAOs) that differentiate high from average performance".

2) But the researches neglect the basic difference in brain which would directly lead to the difference in performance of knowledge work. Try to explore the competency in neurological view will reveal the basic difference exist in individuals.

The common method to study competency is under skepticism [14]. The validity of "competencies" as measurable constructs appears to be at the core of this controversy [15]. Specifically, the process of deriving competencies requires a rather large inferential leap which is too subjective [2]. For BET, which require the employee to tell the most successful and unsuccessful events, may exist the flash effect. And the employee may just tell the most profound part in their memory which may not be the key factors lead to the performance. And some scholars resort to questionnaire or interview which is also subjective.

Beside the traditional method to investigate the competency, there should be some new methods which should be more objective. And using the neurological method will meet the need.

Our research focus on two hypotheses:

1) The nature of the knowledge work is to use mind operation (MO) to process the information. So we could apply the mind operation to represent the work itself.
2) The difference in competency could be reflected in the speed and efficiency of mind operations which would be reflected in brainwaves.

\section{The Confirmatory Research on MO}

\subsection{The Hypothesis for the Concept Model of the MO}

Before classic scientific management, the process of manual movements was a "black box" similar to knowledge tasking. Since Taylor and Gilbreth brought forward the concepts of "basic movement" and "therbligs", manual processes began the "white box" which can be described and measured. This became the foundation of scientific management. If we describe the process of knowledge task by activities, the inner process of it can turn the "black box" into a "white box". In cognitive science, the activity is the mental operations.

Knowledge work is a complex, unstructured and knowledge-intensive process that depends on the human cognitive capacity. Knowledge work can be considered as a process that requires knowledge from both internal and external source to generate information-based products.

The knowledge workers process the information as follows: input, process and output. Newell and Simon put forward the classic model as follows shown in Figure 1.

The processor comprise 3 elements: 1) Elementary information processes, represent, change and compare the symbols; 2) Short memory, keep the basic structure of the symbols; 3) Interpreter, synergize the information and determine the consequence of the information process.

In 1959, Wofac Corporation took the lead to put forward the method of Variable Factor Program (VFP), which studies the brainwork. In 1967, it brought forward Wofac Mento-Fator System (WMFS) based on Predetermined Motion-Time Study (PMTS). WMFS had determined the task element system which included fourteen elements: sight movement, sight watching, looking, nerve transmitting, distinguishing, estimating, identifying, deciding, remembering, recalling, calculating, confirming, transforming and attention transferring.

Guilford brought forth his "three-dimension" theory of intelligence in 1959. This theory considered that intelligence was a three-dimensional space structure comprised of operations (thinking methods, including five components: cognition, memory, divergent thinking, converge thinking, evaluating) $\times$ contents (objects which thinking

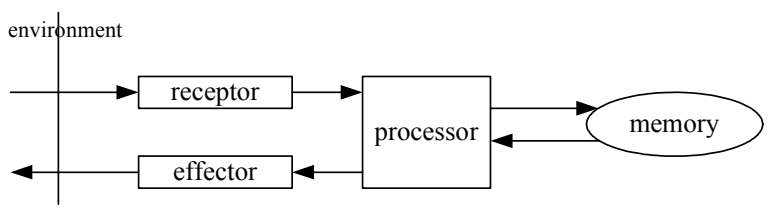

Figure 1. Information process model 
operates on: figure, symbol, semantic meaning, action) $\times$ results (outcomes when certain operation acts on certain content: cell, kind, relation, system, transform, meanings). In the middle of the 1970's, the cognitive revolution began to impact on the research field of intelligence. The most representative research was Sternberg's Triarchic, "Theory of Human Intelligence" and the PASS theory put forward by Das. Their consensus in intelligence research was a transferring focus from traits analysis to interior process. They depicted the mind mechanism of intelligence operation by way of the cognitive process. They used the process analysis method of information-process, and described the interior process of intellective activities more quantitatively.

A component was the basic cell of intelligence operation analysis in Sternberg's Triarchic Theory of Human Intelligence. Sternberg's thought was that a "component is a type of basic information processing. It operates on the basis of interior representation of objects or symbols. Such a process can transform a sensation input into a concept representation; it also can transform a concept representation into a movement output" [16]. Sternberg divided components into metacomponents, performance components and knowledge-acquisition components. Metacomponent was the highest-level control process. It played a role on constituting plans, choosing strategy, supervising execute, appraising results and adjusting feedback. The performance component was the component that a subject used when he implemented task operations with various strategies. Its function was implementing the dictates of metacognition and carrying out various idiographic cognitive process operations. Some performance components would only be used in a multitude of special tasks. Universal performance components, such as coding, combination and comparing of stimulating, inference etc., gained more attention [16]. Those could be viewed as basic mind operations, or mind operations at the "therblig" level.

In light of the analysis from the view of cognitive psychology, we can regard the process of knowledge task as the process of information symbol transformation [17]. In the view of modern cognitive ergonomics, knowledge task is the reasonable sequence of MO [18].

Using the perspective of the Scientific Management's process, the analysis of knowledge task process can be thought of being comprised of "a series of" mind operations which are similar to the "basic motions" or "Therbligs". From the perspective of cognitive science, knowledge task is an operational (transforming) process of the mind on information symbols. The mind's operation is an algorithm of knowledge tasking (transformative rule). Based on these definitions, the mind's operation can be viewed as "operational modes of information processing", while knowledge task process is the set of these information-processing modes (mind operations). We can then present simple manipulative definitions of mind operation concepts on the "therblig" level as follows:

1) Metacognition. Simply speaking, metacognition is cognition and adjustment on cognitive activity processes. It plays a role in constituting plans, choosing strategies, supervising execute, appraising results and adjusting feedback. It is the highest-level control or fountainhead of the following MOs.

2) Distinguishing. The ability to discern and confirm expressive forms of information, every kind of perception, discrimination and recognition. Its meaning is similar to the "cognize" defined by Guilford.

3) Memory. Maintains existing knowledge, searches and memorizes new information.

4) Transforming. Changes the forms of information. For example, transform letter information into table information.

5) Imagining. Changes and transforms images into new one. For example, we read the ancient poetry sentence "Flocks and herds appear as grass bends to wind"; it would appear a beautiful picture in our brain. This is the result of imagination. When we imagine, we all depend on the images already stored in our brain.

6) Character extracting. Breaking down the whole object (cognitive object) into parts and extract the characters of cognitive objects. It has three levels: 1) Action thinking level, such as tearing down the clock's parts one by one within brain; 2) Imagery thinking level, such as breaking down the image of a tree into roots, branches and leaves; 3) Abstract thinking level, such as disassembling the chemistry equation.

7) Character integrating. Combining the parts of the cognitive objects, i.e., characters, attributes, within the brain. "Character integrating" and "character extracting" are both dialectic mind operations. They also have three levels: 1) Motion thinking level, such as assembling the clock's parts one by one within the brain; 2) Imagery thinking level, such as synthesizing the image of roots, branches and leaves into a tree within the brain; 3) Abstract thinking level, such as combining and establishing simultaneous equations.

8) Reasoning. Concluding from known or assumptive facts, or inferring a new judgment from one or more known judgments.

9) Concretization. Applying general conclusions to idiographic things; it is a type of deduction.

\subsection{The Empirical Study of MO}

\subsubsection{The Collection and Summary of the Data}

We used the questionnaire to collect the data to verify the common mind operations that the knowledge workers will use.

The sources of subjects in this study were students in the following programs: Master of Software Engineering (MSE) in University of Electronic Science and Technol- 
ogy of China (UESTC), Master of Business Administration (MBA) in UESTC. 235 questionnaires were distributed and 191 completed questionnaires were returned. In these valid questionnaires, there were $118 \mathrm{MSE}$ and 73 MBA; 143 males', 36 females' and 12 people didn't fill "sex".

The data used SPSS11.5 for statistical processing and LISREL8.53 for confirmatory factor analysis (CFA). We eliminated four items whose least component load was below 0.36. 41 items were processed using LISREL8.53 analysis and the data samples composed by the 191 subjects was processed within the LISREL8.53 - according to the structural equation method.

\subsection{Results and Discussions}

The structure of mind operation concepts set is shown in Figure 2 below.

The program chose fit indices as follows: Chi-square $\left(X^{2}\right)$, NNFI and CFI (critical value is 0.9), RMSEA (critical value is 0.08) [19]. $X^{2}$ goodness-of-fit test is the most often used measure standard about a models degree of total fit. According to the critical values of each index, the data in Table 1 showed that the structural equation model described in Figure 1 fit the observed data; the fit indices were comparatively perfect. In this paper, RMSEA equaled 0.06 and less than 0.08 (RMSEA $=0.06$ $<0.08$ ). The absolute index RMSEA weighed the fit degree between the theoretical model and sample data [19]. This indicated that observed data's ability to interpret knowledge task process with a 9-factors model showed in Figure 2. The knowledge task process of existing nine factors and the structural relation which metacognition controlled the other mind operations was also verified. Knowledge task process can be interpreted

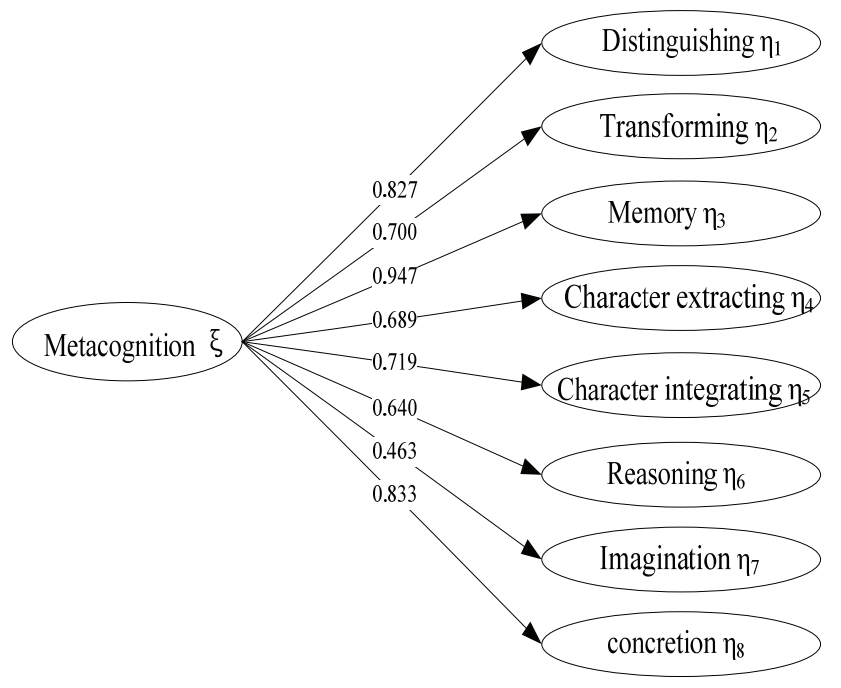

Figure 2. Structure of mind operation concepts set
Table 1. Fit indices of MO concept set

\begin{tabular}{rcccc}
\hline$\chi^{2} / d f$ & NNFI & IFI & CFI & RMSEA \\
\hline 1.669 & 0.916 & 0.924 & 0.924 & 0.0593 \\
\hline
\end{tabular}

by mind operational concept structure showed in Figure 2. The nine factors were comprised of metacogniton and other eight factors controlled by metacognition.

\section{The EEG Study of MO}

During the knowledge work, the workers usually use the MOs to process the information. So the difference in the speed and efficiency of the MOs would eventually lead to the difference in performance. We designed the mental arithmetic problem as the experimental materials. Mental arithmetic is a typical knowledge task which needs most basic cognitive functions to be achieved, such as attention, computing, memory et al.

\subsection{The Design of the Experiment}

Using the 17 inches computer screen to display the problems. The problem appears in the center of the screen. The four choices appear 2 centimeters above bottom. The distance from the eye to the screen is about 40 centimeters. Keep the eye horizontal with the center of the screen and the sight vertical with the screen. 15 college males are as the subjects. All short hair considered the requirement of electroencephalogram. Good eyesight, right handed and without brain damage. Using the EGI system 200 made from American Electrical Geodesics Incorporated to record the data. Through 128 channels to collect sample and the frequency is $500 \mathrm{~Hz}$; the NetAmps 200 and 129 lead electrode hat collected the brain electric signal. The precision of reaction time is $0.001 \mathrm{sec}$. The subjects are asked to finish the 10 mental arithmetic problems as 14 digits to add.

\subsection{The Result and Analysis}

\subsubsection{Divided the Subject According to the Reaction Time}

Reaction time is a popular index used in experimental psychology. It could measure the activation and restrain of the pallium, and further used to analyze the mental activities, such as sense, attention, learning, thinking etc..

To delete the data that is abnormal and beyond the 3 times standard deviation, and analyze the data by SPSS 15.0, here is the result:

The average reaction time of the quick group is $21292.438 \mathrm{~ms}$; the average reaction time of the slow group is $27776.494 \mathrm{~ms}$. There exist significant difference in reaction time. $(t=3.407, P=0.009<0.05)$

\subsubsection{Computed EEG Topography (CET)}

Computed EEG Topography (CET) also named as EEG Cartography, Brain Electrical Activity Mapping, EEG Isopotential Mapping. Put the data into matlab2006, and use the toolbox of EEGLAB. Here is the result: 


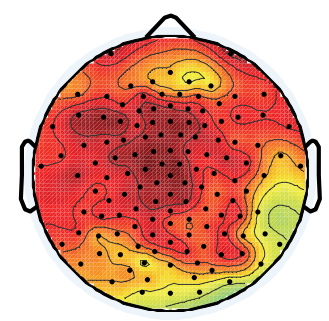

Figure 3. Quick group

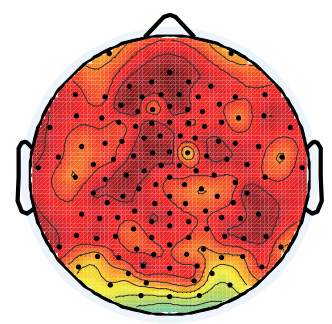

Figure 4. Slow group

The brain could be divided into four areas: frontal lobe, parietal lobe, occipital lobe and temporale lobe. And each one is in charge of the specific function: parietal lobe is relevant with the visual processing. Frontal lobe is relevant with the action, direction, computation and discerning. Temporale lobe is relevant with the sound, verbal comprehension and memory. Frontal lobe is relevant with the thinking, concept, the execution of plan and conscientious emotion.

Compare the two group's CET, we can tell there exist difference in activation of the different brain areas. During the mental arithmetic, most areas were involved. A lot research indicates that during the task with number, the parietal lobe will be activated. The meta analysis of activation images point out that the HIPS (horizontal segment of intraparietal sulus) is the area which represent the number [20]. Our research supports the finding. The difference between the quick and slow group is that the frontal lobe of quick group is activated in higher degree. The frontal lobe connects with the working memory. The different performance in mental arithmetic is probably due to the involvement of the meta-cognition of the working memory.

\subsubsection{Brain Electric Power Spectrum Analysis}

The electroencephalogram (EEG) during information processing is influenced by specific changes in brain electrical activity. Brain electric power spectrum analysis is one quantitative analysis developed recently. It could reflect the frequency characteristic of brain electrical signals, and also could reflect the density of brain

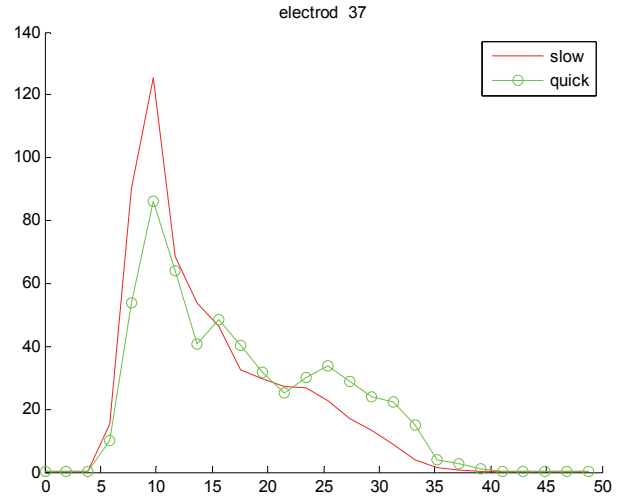

Figure 5. The PSD of electrode 37

electrical signals. The basis of brain electric power spectrum analysis is power spectrum density (PSD).

We use the EEGLAB and Matlab2006 to further explore the data of electroencephalogram. Compare the common activated area of the two groups, and choose the electrode 37 to be analyzed, the result is shown in Figure 5.

We can tell there exist difference in $\alpha(8 \sim 12.8 \mathrm{~Hz})$. The frequency band of quick group is lower than the slow group. A lot of researches indicate the power of $\alpha$ is relevant with the IQ [21,22]. Jausovec [23,24] also indicated that the differences in EEG current density related to intelligence.

\section{Conclusions}

The essence of knowledge work is the information process. And the process could be represented by 9 common mind operations. The competency difference between the employees could partly be explained as the difference in speed and efficiency of the mind operation. So explore the competency of the knowledge workers in neurological method will help to give the full picture of the competency. And the neurological method also could be more objective to study the competency. And the conclusion will be more convincible. The neurological method has a bright future in the field of economics and management.

\section{REFERENCES}

[1] A. D. Lucia and R. Lepsinger, "The art and science of competency models," Jossey-Bass, San Francisco, 1999.

[2] J. S. Schippman, R. A. Ash, M. Battista, L. Carr, D. Eyde, and B. Hesketh, et al., "The practice of competency modeling," Personnel Psychology, Vol. 53, pp. 703-740, 2000.

[3] M. A. Armstrong, "Handbook of personnel management practice," Kogan Page, London, 1991.

[4] W. F. Cascio, "Whither industrial and organizational psychology in a changing world of work," American Psychologist, Vol. 50, pp. 928-939, 1995. 
[5] G. R. Ferris and T. A. Judge, "Personnel/Human resources management: A political influence perspective," Journal of Management, Vol. 17, pp. 447-448, 1991.

[6] L. F. Zhong and K. Shi, "The new development of research of competencies," Nankai Business Review, Vol. 2, pp. 4-8, 2003.

[7] D. C. McClelland, "Testing for competence rather than for intelligence," American Psychologist, Vol. 28, pp. $1-4,1973$.

[8] K. Shi, J. Wang C, and C. P. Li, "Assessment on competency model of senior managers," Acta Psychological Sinica, Vol. 34, No. 3, pp. 306-311, 2002.

[9] L. F. Zhong and K. Shi, "The competency model of senior managers in Chinese family firms," Acta Psychological Sinica, Vol. 36, No. 1, pp. 110-115, 2004.

[10] E. Folch-Lyon and J. F. Trost, "Conducting focus group sessions," Studies in Family Planning, Vol. 12, pp. 443449, 1981.

[11] S. Jorgen, "Understanding human competence at work: An interpretative approach," Academy of Management Journal, Vol. 42, No. 1, pp. 9-25, 2000.

[12] W. S. Chen, "Study on the competency model for human resource administrators in Chinese companies," The Doctor Degree Thesis of the Xiamen University, 2004.

[13] X. Z. Luo and D. M. Miao, "A research on the coherence of competency model ratings," Psychological Science, Vol. 27, No. 5, pp. 1192-1194 (in Chinese), 2004.

[14] T. R. Athey and M. S. Orth, "Emerging competency methods for the future," Human Resource Management, Vol. 38, No. 3, pp. 215-226, 1999.

[15] E. Lawler, "Competencies: A poor foundation for the new pay," Compensation and Benefits Review, Vol. 28, No. 6, pp. 20-22, 1996.

[16] R. J. Sternberg, "Beyond IQ: A triarchic theory of human intelligence," Cambridge University Press, New York, 1985.

[17] H. A. Simon, "Search and reasoning in problem solving," Artificial Intelligence, Vol. 21, pp. 7-29, 1983.

[18] J. H. Wang, "Military ergonomics," Beijing: Defense University Press, 1997.

[19] Z. L. Wen, K. T. Hau, and W. M. Herbert, "Structural equation model testing: Cutoff criteria for goodness of fit indices and Chi-square test," Acta Psychological Sinica, Vol. 36, No. 2, pp. 186-194, 2004.

[20] S. Dehaene, M. Piazza, P. Pinel, and L. Cohen. "Three parietal circuits for number processing," Cognitive Neuropsychology, Vol. 20, pp. 487-506, 2003.

[21] M. Doppelmayr, W. Klimesch, W. Stadler, D. Pöllhuber, and C. Heine, "EEG alpha power and intelligence," Intelligence, Vol. No. 30, pp. 289-302, 2002.

[22] R. G. Schmid, W. S. Tirsch, and H. Scherb, "Correlation between spectral EEG parameters and intelligence test variables in school-age children," Clinical Neurophysiology, Vol. 113, No. 10, pp. 1647-1656, 2002.

[23] N. Jausovec "Differences in cognitive processes between gifted, intelligent, creative and average individuals while solving complex problems: An EEG study," Intelligence, Vol. 28, pp. 213-237, 2000.

[24] N. Jausovec and J. Ksenija, "Differences in EEG current density related to intelligence," Cognitive Brain Research, Vol. 12, pp. 55-60, 2001. 